\title{
Optical Projection Tomography Technique for Image Texture and Mass Transport Studies in Hydrogels Based on Gellan Gum
}

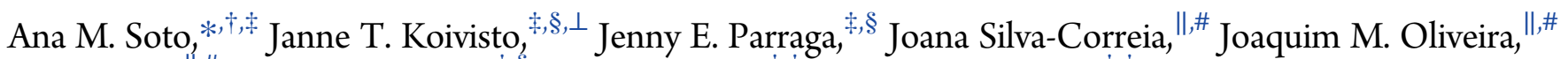

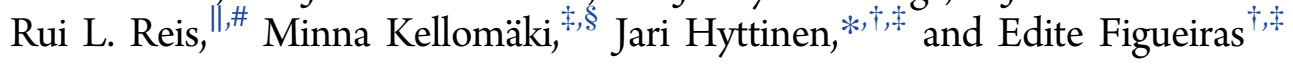 \\ ${ }^{\dagger}$ Computational Biophysics and Imaging Group, ELT Department, Tampere University of Technology, 33720 Tampere, Finland \\ ${ }^{\ddagger}$ BioMediTech - Institute of Biosciences and Medical Technology,33720 Tampere, Finland \\ ${ }^{\S}$ Biomaterials and Tissue Engineering Group, ELT Department, Tampere University of Technology, 33720 Tampere, Finland \\ ${ }^{\perp}$ Heart Group, BioMediTech, University of Tampere, 33720 Tampere, Finland \\ "3B's Research Group, Biomaterials, Biodegradables and Biomimetics, University of Minho, Headquarters of the European Institute of \\ Excellence on Tissue Engineering and Regenerative Medicine Barco GMR, 4704-553 Braga, Portugal \\ \#ICVS/3B's - PT Government Associate Laboratory, 4710-057 Braga/Guimarães, Portugal
}

\section{Supporting Information}

ABSTRACT: The microstructure and permeability are crucial factors for the development of hydrogels for tissue engineering, since they influence cell nutrition, penetration, and proliferation. The currently available imaging methods able to characterize hydrogels have many limitations. They often require sample drying and other destructive processing, which can change hydrogel structure, or they have limited imaging penetration depth. In this work, we show for the first time an alternative nondestructive method, based on optical projection tomography (OPT) imaging, to characterize hydrated hydrogels without the need of sample processing. As proof of concept, we used gellan gum (GG) hydrogels obtained by several cross-linking methods. Transmission mode OPT was used to analyze image microtextures, and emission mode OPT to study mass transport. Differences in hydrogel structure related to different types of cross-linking and between modified and native GG were found through the acquired Haralick's image texture features followed by multiple discriminant analysis (MDA). In mass transport studies, the mobility of FITC-dextran (MW 20, $150,2000 \mathrm{kDa}$ ) was analyzed through the macroscopic hydrogel. The FITC-dextran velocities were found to be inversely proportional to the size of the dextran as expected. Furthermore, the threshold size in which the transport is affected by the hydrogel mesh was found to be $150 \mathrm{kDa}$ (Stokes' radii between 69 and $95 \AA$ ). On the other hand, the mass transport study allowed us to define an index of homogeneity to assess the cross-linking distribution, structure inside the hydrogel, and repeatability of hydrogel production. As a conclusion, we showed that the set of OPT imaging based material characterization methods presented here are useful for screening many characteristics of hydrogel compositions in relatively short time in an inexpensive manner, providing tools for improving the process of designing hydrogels for tissue engineering and drugs/cells delivery applications.

\section{INTRODUCTION}

Tissue engineering (TE) is an interdisciplinary field which aims to develop technologies able to restore, replace and regenerate damaged tissue or organs. ${ }^{1}$ Despite the advances in TE, the current medical treatments are often not optimal, as many of the replaced tissues or prostheses do not function as well as healthy tissue or they can trigger severe immunological response. To overcome many of these problems, new cellular strategies and sophisticated biomaterials are being developed, together with new tools to study their behavior. ${ }^{1-3}$

The main strategy used in TE is to combine a biomaterial scaffold with living cells and bioactive molecules, which together are able to develop a biological process like differentiation and regeneration. ${ }^{1}$ To achieve this goal, it is necessary to develop scaffold materials which resemble the natural cellular environment and provide the cells an adequate support for growth. ${ }^{4}$ Among the biomaterials, hydrogels have emerged as promising materials for TE due to their structural resemblance to the extracellular matrix (ECM), biocompatibility and tunable properties, such as biodegradability, permeability to nutrients and waste products, injectability and mechanical characteristics. ${ }^{5-7}$ Hydrogels are hydrophilic polymeric networks with high content of water which can be obtained from natural sources (i.e., alginate, chitosan, gellan

Received: March 1, 2016

Revised: $\quad$ May 2, 2016

Published: May 3, 2016 
Table 1. Gellan Gum Hydrogels Fomulations and Preparation Methods

\begin{tabular}{|c|c|c|c|c|c|}
\hline & abbreviation & polymer & cross-linker & $\begin{array}{l}\text { final cross-linker } \\
\text { concn }\end{array}$ & cross-linking method \\
\hline \multirow[t]{3}{*}{ group 1} & iGG & low acyl gellan gum $2 \% \mathrm{w} / \mathrm{v}$ & PBS $1 \times$ & $10 \% \mathrm{v} / \mathrm{v}$ & ionic (physical) \\
\hline & iGG-MA & $\begin{array}{l}\text { methacrylated gellan gum } \\
2 \% \mathrm{w} / \mathrm{v}\end{array}$ & PBS $1 \times$ & & ionic (physical) \\
\hline & photoGG-MA & $\begin{array}{l}\text { methacrylated gellan gum } \\
2 \% \mathrm{w} / \mathrm{v}\end{array}$ & $\begin{array}{l}\text { MBF } 98 \%(w / v)+ \\
\text { PBS } 1 \times\end{array}$ & MBF $0.1 \%(w / v)$ & $\begin{array}{l}\text { ionic and UV photo-cross-linking (physical and } \\
\text { chemical) }\end{array}$ \\
\hline \multirow[t]{3}{*}{ group 2} & iGG-SPM-H & gellan gum $0.5 \% \mathrm{w} / \mathrm{v}$ & spermine $1.79 \mathrm{mM}$ & $0.24 \mathrm{mM}$ & ionic (physical) \\
\hline & iGG-SPM-L & gellan gum $0.5 \% \mathrm{w} / \mathrm{v}$ & spermine $0.90 \mathrm{mM}$ & $0.12 \mathrm{mM}$ & ionic (physical) \\
\hline & iGG-Ca & gellan gum $0.5 \% \mathrm{w} / \mathrm{v}$ & $\mathrm{CaCl}_{2} 10 \mathrm{mM}$ & $2.5 \mathrm{mM}$ & ionic (physical) \\
\hline
\end{tabular}

gum) or synthetic sources (i.e., poly(ethylene oxide) (PEO), poly(vinyl alcohol) (PVA), and poly(acrylic acid) (PAA) $)^{8}$

Hydrogels have been used for several TE applications ${ }^{9-14}$ (e.g., cartilage, intervertebral disc (IVD), meniscus, peripheral nerve, and cardiac) as these materials can be processed under mild conditions, making cells and drugs encapsulation a very straightforward process. In spite of the relatively successful clinical application of various engineered tissues using hydrogel scaffolds, one significant challenge associated with the use of hydrogels is the evaluation of their microstructure and permeability, which are critical factors for cell penetration and survival and tissue regeneration. These factors affect the availability, among others, of nutrients and growth factors; in addition, it is known that there is a natural gradient of nutrients and metabolites within the hydrogels. ${ }^{3,15}$ Bearing in mind that the success of a TE therapy depends of these features, it is important to evaluate the scaffold capacity to transport these biomolecules. $^{15,16}$ Additionally, the determination of the diffusion of drug molecules within the hydrogel is essential for drug delivery applications. Thus, parameters such as polymer network mesh size, porosity and pore interconnectivity are key elements since they are important not only for cell nutrition, but also for cell adhesion, migration and proliferation, differentiation, signaling, and vascular growth. ${ }^{4,16}$

Transport of molecules in hydrogels occurs between the polymer chains in water-filled regions. The movement of the molecules is limited by the polymer network density, the mobility of the polymer chains and the presence of charged groups that potentially could bind biological molecules. ${ }^{17}$ Consequently, the movement of molecules is reduced when the cross-linking density increases and the volume fraction of water of the hydrogel decreases. ${ }^{18}$

When developing new hydrogels, the study of porosity and mass transport properties in physiologically relevant conditions is one the most critical design points. ${ }^{3,19}$ Conventionally available imaging methods able to characterize hydrated hydrogels have many limitations. They require sample processing or drying, which can change the hydrogel structure (once a hydrogel is dried, the molecular polymer mesh may collapse into thicker walls of pores and therefore the smaller pores are expected to shrink and large ones become even larger), are destructive, or have limited imaging depth penetration. For instance, scanning electron microscopy (SEM) is a destructive method, as it requires sample drying and electrically conductive coating, is limited to the surface of the material with a penetration depth smaller than $200 \mathrm{~nm},{ }^{20,21}$ and only gives morphological information on a dried sample surface, ${ }^{20}$ which can be different from the wet conditions. On the other hand, X-ray microcomputed tomography $(\mu \mathrm{CT})$ is able to image the three-dimensional structure of the hydrogels, but it also requires sample drying or contrast agents or both. ${ }^{20}$
Confocal microscopy (CM), two-photon fluorescence microscopy (TPFM), and optical coherence tomography (OCT) offer the possibility of $3 \mathrm{D}$ imaging of hydrated hydrogels. However, $\mathrm{CM}$ and TPFM are confined to fluorescent signals, require sample labeling or autofluorescence, and they are focused in the microscopic scale (CM and TPFM imaging depth is smaller than $100 \mu \mathrm{m}$ and $1 \mathrm{~mm}$, respectively). In addition, TPFM can produce thermal damage to the sample. ${ }^{20,22}$ OCT is able to generate 3D images but the size of the specimen is limited by the penetration depth of less than $2 \mathrm{~mm} .{ }^{20}$ In addition, these techniques can be time-consuming and costly. ${ }^{20}$

In this work, we propose an alternative method to characterize hydrogels based on optical projection tomography (OPT). OPT is a nondestructive three-dimensional microscopy technique originally developed by Sharpe et al. in $2002^{23}$ to image the anatomy of embryos and small organs. OPT can be used to image fluorescent (emission mode) and nonfluorescent (transmission mode) specimens embedded in a refractive index matching solution. The ability to image samples in the mesoscopic scale opens the possibility to image complete intact specimens smaller than $10 \mathrm{~mm}^{24}$ or alive samples. ${ }^{24,25}$ However, for opaque specimens, optical clearing of the sample is required for depth imaging. The optical clearing protocols are toxic, making in vivo imaging a challenge for most of the biological samples. On the other hand, due to the transparency of most hydrogels, clearing protocols are not required for OPT imaging. $^{26}$

In the present work, we aim to show for the first time how OPT can offer methods to characterize hydrogel structure and its properties, expanding the preliminary results by Figueiras et $\mathrm{al}^{27}$ and Soto and co-workers. ${ }^{26,28}$ Here we demonstrate the characterization of gellan-gum-based hydrogels by analyzing image microtextures and mass transport properties. This is done to identify differences between hydrogels structure according to their cross-linking method based on OPT imaging. Specifically, we highlight that OPT is a useful method for imaging transparent hydrogels without the need of labeling, drying, or other sample processing. The use of 3D reconstruction algorithms offers the possibility to estimate/ recover the whole $3 \mathrm{D}$ volume of hydrated hydrogels. Image texture analysis enables one to perform a comparative study of newly developed gellan gum hydrogel compositions. Finally, we show that OPT offers the possibility to image the transport of molecules through the 3D hydrogel scaffold in real time using OPT in the emission mode.

\section{MATERIALS AND METHODS}

2.1. Preparation of Hydrogels. In this work, we analyzed two different groups of gellan gum (GG) hydrogels. Table 1 describes the composition and cross-linking method used in each type of hydrogel. The first group includes hydrogels based on natural or methacrylate- 
modified GG (GG-MA) prepared by ionic and/or photo-cross-linking method. The second group includes physically cross-linked gellan gum with the bioamine spermine (SPM), at two different concentrations, 0.24 and $0.12 \mathrm{mM}$, described as iGG-SPM-H and iGG-SPM-L, respectively. In addition, it was used a reference hydrogel cross-linked with calcium ions (iGG-Ca). The first group of hydrogels have been used for intervertebral disc (IVD) tissue engineering applications, ${ }^{9}$ and the second group was developed for neural and cardiac cell culture disease modeling applications. ${ }^{29}$

All reagents were purchased from Sigma-Aldrich, unless otherwise specified. All the hydrogel solutions were cast in fluorinated ethylene propylene (FEP) tubes with $2 \mathrm{~mm}$ inside diameter (Adtech Polymer Engineering, U.K.) for transmission OPT imaging and in $5 \mathrm{~mL}$ polystyrene cuvettes (BRAND UV cuvette, Sigma-Aldrich) for emission OPT imaging.

iGG, iGG-MA, and photoGG-MA with UV photo-cross-linker were prepared as described by Silva-Correia and co-workers. ${ }^{9,30}$ The iGG hydrogels were prepared by dissolving low acyl gellan gum (GG) (Gelzan CM) powder in distilled water under constant agitation to obtain a $2 \% \mathrm{w} / \mathrm{v}$ solution. The solution was heated progressively to 90 ${ }^{\circ} \mathrm{C}$ maintaining this temperature for $30 \mathrm{~min}$. Then, the temperature was gradually decreased to $60-65{ }^{\circ} \mathrm{C}$ and phosphate-buffered saline (PBS, pH 7.4) was added to a final concentration of $10 \% \mathrm{v} / \mathrm{v}$ in GG solution. The temperature was continuously decreased to $50{ }^{\circ} \mathrm{C}$ and poured in the corresponding container. Then, the hydrogels were allowed to cross-link and stabilized by immersion in PBS.

Both iGG-MA and photoGG-MA hydrogels were prepared by using methacrylated gellan gum (GG-MA) solution at $2 \%(\mathrm{w} / \mathrm{v})$. GG-MA was dissolved in distilled water under vigorous agitation at room temperature until complete and homogeneous dissolution of the powder. For preparing iGG-MA, the solution was cast into its corresponding container and immersed in PBS ( $\mathrm{pH} 7.4$ ) overnight. For preparing photoGG-MA, the photoinitiator methyl benzoylformate $(\mathrm{MBF}, 98 \%)$ was added to the solution to a final concentration of $0.1 \%(\mathrm{w} / \mathrm{v})$. The gel was transferred to the corresponding container and the hydrogels were obtained by exposure to ultraviolet (UV) light (366 nm; UV lamp Triwood 6/36, Italy). The hydrogels prepared in FEP tubes were exposed for $10 \mathrm{~min}$, whereas the hydrogels prepared in the cuvettes were exposed to UV light for a total period of $40 \mathrm{~min}$, i.e., $10 \mathrm{~min}$ for each face of the cuvette. Bioamine based hydrogels, i.e. GGSPM-H and GG-SPM-L, were prepared using spermine tetrahydochloride (SPM), as cross-linker agent. Briefly, the hydrogel samples were prepared by dissolving GG powder in a deionized water solution of $10 \%(\mathrm{w} / \mathrm{v})$ sucrose to obtain a concentration of $0.5 \% \mathrm{w} / \mathrm{v}$. The SPM was dissolved in $10 \%$ sucrose and diluted to $1.79 \mathrm{mM}$ (iGGSPM-H) and $0.90 \mathrm{mM}$ (iGG-SPM-L) concentrations. All solutions were sterile filtered at $60{ }^{\circ} \mathrm{C}$ with $0.8 / 0.2 \mu \mathrm{m}$ Acrodisc syringe filter, PALL Corporation, before the gelation process. When preparing the gel samples, the solutions were first heated in water bath at $37{ }^{\circ} \mathrm{C}$. SPM and GG were mixed in a volume ratio of $4: 25$, cast in their corresponding containers and stored overnight at RT before imaging.

Ca-GG hydrogels were prepared in a similar way as previous described for the bioamine-based hydrogels. GG and $\mathrm{CaCl}_{2}$ (Honeywell Riedel-de Haën, Germany) were dissolved in $10 \%$ sucrose at $0.5 \%$ $\mathrm{w} / \mathrm{v}$ and $10 \mathrm{mM}$, respectively. The solutions were sterile filtered similarly and the GG solution and $\mathrm{CaCl}_{2}$ solution were heated in a water bath to $37{ }^{\circ} \mathrm{C}$ before the gelation process. The solutions were mixed in a volume ratio of 1:3 with final cross-linker concentration of $2.5 \mathrm{mM}$. The hydrogels were cast in corresponding container and stored overnight at RT before imaging.

2.2. Transmission OPT Imaging for Image Texture Analysis. An OPT system as previously described ${ }^{27}$ with transmission and emission modes was used. A scheme of the system is shown in Figure 1. Five samples of each type of hydrogel were imaged using OPT transmission mode. The hydrogels were prepared into FEP tubes as described above and submerged in water. Projections images were taken around entire $360^{\circ}$ at steps of $0.9^{\circ}$ resulting in 400 images. The images were taken with a $5 \mathrm{x}$ objective, providing a resolution of about $3 \mu \mathrm{m}$. The 3D images of the samples visualizing the 3D internal structures we reconstructed from the projection images using back

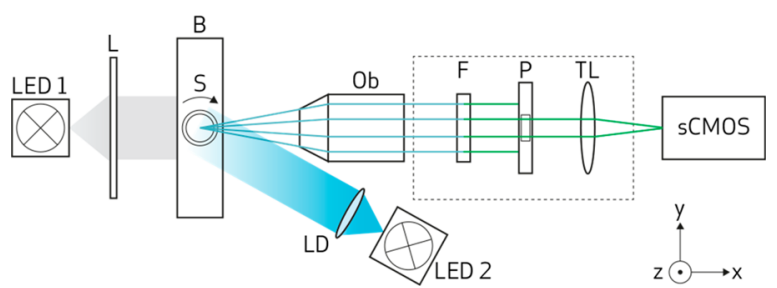

Figure 1. Schematic diagram of the OPT setup: hydrogel samples inserted in FEP tubes are rotated in the rotation stage (S) inside a water bath (B). For brigth field illumination a white light (LED 1) and a telecentric lens (L) are used. For fluorescence illumination, $470 \mathrm{~nm}$ wavelenght (LED2) collimated with a lens with diffuser (LD) are used. The detection system consist of objective lens $(\mathrm{Ob})$, a band-pass filter (F) used only for fluorescence imaging, a pinhole (P), a tube lens (TL), and a sCMOS camera.

projection algorithm as previously described. ${ }^{27}$ Supporting Information Video 1 shows two examples of hydrogels: (a) iGG-SPM-H projections, (b) the reconstruction of (a), (c) photoGG-MA projections, and (d) reconstruction of (c).

2.3. Image Processing and Texture Analysis. Image texture analysis has been carried out using multiple discriminant analysis (MDA). (Note: Image texture analysis used in this work should not be confused with mechanical texture analysis performed in materials science and food industry. ${ }^{41}$ ) The analysis was performed to assess and compare the microscopic structures of the hydrogels in the projection images and in their 3D reconstructions using Haralick's textural features ${ }^{31}$ in the five samples of each hydrogel type. Preprocessing of the projection images included flat field correction of the illumination nonuniformity created by the white LED (LED1, Figure 1) using 2D polynomial fitting, and removal of the tube from the images by manual segmentation. Histogram equalization was applied to the projections and slices images before performing the textural analysis. The gray level co-occurrence matrix (GLCM), also known as spatial gray level dependence matrix (SGLD), was computed for each image using one and three pixels length at different directions, i.e., $0^{\circ}, 45^{\circ}, 90^{\circ}$ and $135^{\circ}$, respectively. From the calculated co-occurrence matrix of each image, 13 textural features were computed. ${ }^{31}$ Due to the high dimensionality of Haralick's features, MDA was used to reduce dimensionality and find differences between textural features of the hydrogels. MDA is a method used to project the feature space onto a lower dimension space in such a way that maximizes the interclass scatter and minimizes the intraclass scatter. ${ }^{32}$

2.4. Emission OPT Imaging for Mass Transport Studies. The hydrogel samples $(\sim 1 \mathrm{~mL})$ for mass transport studies were prepared as described above in $5 \mathrm{~mL}$ polystyrene cuvettes. FITC-Dextran (TdB Consultancy AB, Sweden) with different molecular weights, i.e., 20, 150 , and $2000 \mathrm{kDa}$ (emission $520 \mathrm{~nm}$, absorbance $493 \mathrm{~nm}$ ), were used to characterize the transport of molecules. Each FITC-Dextran solution was prepared by dissolving the powder in distilled water at a concentration of $10 \mathrm{mg} / \mathrm{mL}$. Each type of FITC-dextran molecule experiments was replicated five times.

For imaging the mass transport, OPT fluorescent projections were taken always in the same angular position of the sample using the OPT in emission mode as represented in Figure 1. For the fluorescence mode a collimated LED of $470 \mathrm{~nm}$ (M470L3, Thorlabs) (LED 2 in Figure 1) was used to excite the samples and a band-pass filter with center wavelength of $520 \mathrm{~nm}$ (EO 67-030, Edmund Optics) was added to the detection system ( $\mathrm{F}$ in Figure 1 ).

The experiments were performed as follows: (a) the focal plane of the imaging system was placed approximately in the middle plane of the cuvette at the upper part of the hydrogel; (b) $100 \mu \mathrm{L}$ of solution of one type of molecular weight FITC-dextran was pipetted to the surface of the sample; and (c) projections were acquired each minute for $4 \mathrm{~h}$ with an exposure time of $0.04 \mathrm{~s}$. The first image was taken before pipetting the FITC-Dextran $(20,150$, or $2000 \mathrm{kDa})$ on the top of the samples. The imaged area consisted of $3 \mathrm{~mm}$ length-square. 

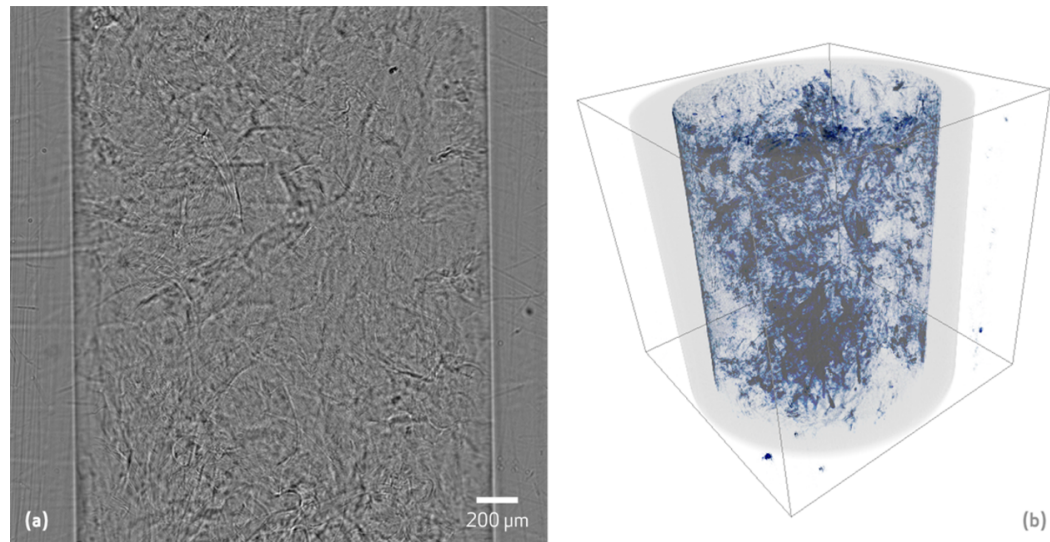

Figure 2. OPT images acquired in transmission mode of a gellan gum hydrogel cross-linked with $\mathrm{Ca}^{2+}$ : (a) projection image and (b) 3D reconstruction.

2.5. Mass Transport Image Processing and Analysis. The data was preprocessed by subtracting the background to remove common elements in all images, such as nonuniformity produced by the LED. This was performed by subtracting the first image acquired, before the addition of the FITC-dextran molecules. We devised a new method to assess the homogeneity of the hydrogels by defining an index of homogeneity. The acquired images were first binarized by Otsús method, ${ }^{33}$ a heuristic method which iteratively calculates the optimum threshold automatically based on the histogram of the image. This threshold value was calculated for each sample. Thus, when the gray level intensity of the pixel is higher than the calculated threshold, the value of the pixel is converted to 1 (white). By its turn, if the value of the pixel is inferior to that for the calculated threshold, the pixel value is converted to 0 (black). This produces a wavefront descending through the image (see Supporting Information Video 2). We assume that when the binarized signal from FITC-dextran does not travel uniformly, the hydrogel is less homogeneous than when traveling uniformly (see Supporting Information Video 3). The index of homogeneity was defined as the measure of the smoothness of the wavefront descending through the hydrogel. To calculate the index of homogeneity, the gradient in the black and white interface was calculated. The gradient points toward the direction of greatest change in every pixel, thus by analyzing the gradient interface it is possible to determine the flatness of said interface. In this manner, when the interface between black and white pixels is flat, the angle of the gradient vector is $90^{\circ}$ (pointing downward in the image). On the contrary, if the interface is not flat, i.e., irregular, its gradient will also be irregular and the angles will be different than $90^{\circ}$ (will point in random directions other than downward). Motivated by this behavior, the index of homogeneity is calculated as the ratio between the total number of $90^{\circ}$ angles and the total number of angles. When the value of the index is closer to 1 , a greater number of $90^{\circ}$ angles is present and thus indicates a flat interface. The binary images were also used to analyze the position of the wavefront at different times giving us the velocity of the transport. We calculated the velocities of the wavefront at time ranges of $50 \mathrm{~min}$ by polynomial fitting of first degree.

Image processing and analysis of all the experiments was performed using MATLAB. The data normality and homoscedasticity were verified using Shapiro-Wilk and Levene statistics, respectively. The data did not show a normal distribution, so the nonparametric MannWhitney $U$ test was used for comparing the differences between groups. Analysis was performed with SPSS v13.0 for Windows (SPSS Inc., Chicago, IL).

\section{RESULTS AND DISCUSSION}

Several hydrogels were obtained through different cross-linking methods as showed in Table 1. During the preparation, the gelation conditions were controlled to reduce variability between samples. Finally, we obtained hydrogels according to the previously published information. ${ }^{9,29,30}$ SPM cross-linked hydrogels appeared more transparent than the MA hydrogels when imaged with OPT. The observed different optical properties were subsequently studied in more detail by image texture analysis.

3.1. Image Texture Analysis. Two different groups of hydrogels were analyzed: the first group includes hydrogels with different cross-linking methods and the second group includes hydrogels with different physical cross-linking agents and concentrations, as showed in Table 1 .

Image texture analysis was performed in the projections acquired with transmission OPT and in the computed reconstructed slices. Figure 2 shows an example of one projection image (Figure 2a) and 3D reconstruction (Figure 2b) of iGG-Ca. The projection image shows the walls of the tube, which were removed for calculating the Haralick's features in both projections and $3 \mathrm{D}$ reconstructions.

From the point of view of image processing, texture is the spatial arrangement of pixel intensities. ${ }^{34}$ The images/ projections acquired by OPT transmission mode shows the contrast caused by transmitted and absorbed light in the sample. This interaction of light with the sample produces spatial variations of intensity in the images, which are known as image texture. By applying filtered back-projection algorithm we reconstructed the $3 \mathrm{D}$ volume of the sample to obtain the spatial distribution of the hydrogels structures; see Figure $2 \mathrm{~b}$ and Supporting Information Video 1. Different spatial characteristics of the internal structure of the samples, such as density variations, created different textures in the images.

Our group previously reported ${ }^{26,27}$ the statistical analysis of hydrogel projections and their $3 \mathrm{D}$ reconstructions. Kurtosis, a measure of the shape of the probability distribution of the image histogram, and entropy, a measure of the randomness of the pixels' intensities, were calculated from the projections and reconstructed slices. It was observed that kurtosis decreases with the increase of image microstructures while the opposite happened for entropy. However, the information derived from the histograms alone is not enough to characterize the texture of the hydrogels, since it does not give information about the spatial distribution of the pixel intensities. ${ }^{34}$ The GLCM is one of the methods used to extract information about the spatial dependence of gray level values and therefore can be used to characterize textures. ${ }^{34}$ It should be noted that the use of the GLCM does not describe shape properties, thus this method is 

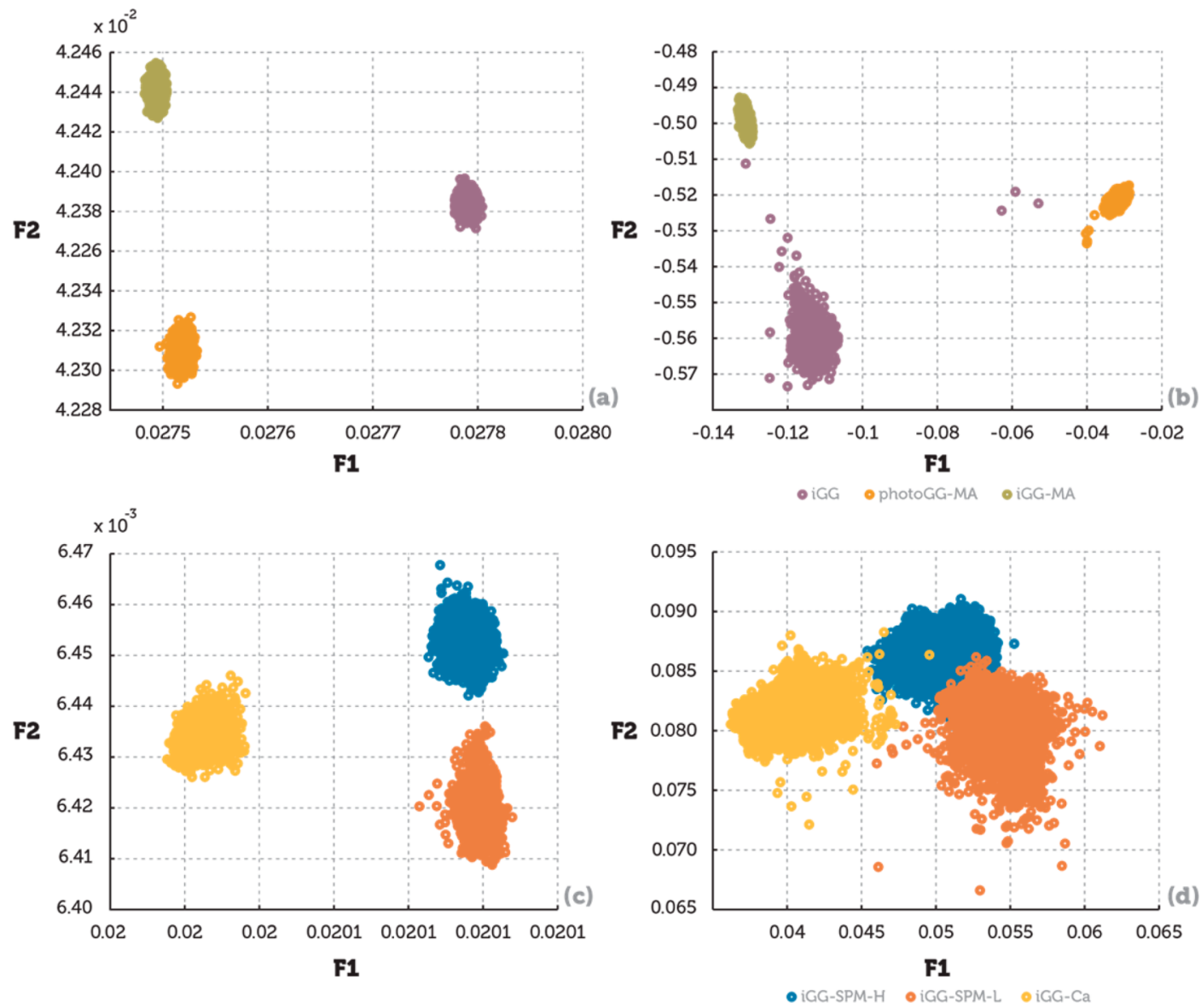

Figure 3. Visualization of the classes projected onto the $2 \mathrm{D}$ feature subspaces created by MDA: (a) OPT projection images of hydrogels from group 1; (b) reconstructed slices of hydrogels from group 1; (c) OPT projection images of hydrogels from group 2; and (d) reconstructed slices of hydrogels from group 2. iGG, ionic cross-linked gellan gum; iGG-MA, ionic cross-linked methacrylated gellan gum; photoGG-MA, photo-crosslinked methacrylated gellan gum; iGG-SPM-L, bioamine low cross-linked gellan gum; iGG-SPM-H, bioamine high cross-linked gellan gum; and iGG-Ca, calcium ions cross-linked gellan gum.

more suitable for characterizing microtextures and not for large scale structures. $^{34}$

By analyzing the textural features of the projections and reconstructed images we quantified and compared different hydrogels, Table 1. The textural analysis tool used (Haralick textural features) provides high dimensional data, and in order to better visualize, understand and compare we used MDA to reduce the dimensionality of the feature space. MDA minimize the high dimensionality of this feature space in a manner that best discriminates between different classes (and thus discriminates between different hydrogels), while minimizing the intraclass variations (minimizing the differences within the same hydrogel samples). Thus, MDA selects the combination of textural features that better characterize the hydrogels. If there were not any significant differences between the hydrogels types, MDA would not be able to separate them in different distinct clusters (Figures 3 and 4). Thus, there are discernible differences in the hydrogels image texture, which is related to their internal spatial structure.

Figure 3 shows the results of the MDA of the projections and reconstruction slices of the two hydrogel groups. The high dimensionality of Haralick's features was reduced to two dimensions with two discriminant functions (F1 and F2), which are built as the linear combinations of Haralick's features that contribute most to the separation between hydrogel classes. The features that contribute the most in projections are
Haralick energy, Haralick entropy and Haralick information measure of correlation $1^{31}$ at different pixel lengths and directions, but these image analysis features should not be confused with their counterparts in chemistry. These features are known to be independent of the different gray intensities. ${ }^{31}$ On the other hand, the other Haralick's features at the different pixel lengths and directions make a stronger contribution to the discriminant functions built by the MDA for the reconstructed slices. It should be noted that the discriminant functions are different for every case.

The MDA of the hydrogels from group 1, shows three distinguishable clusters in the projection images and reconstructed slices, as can be seen in Figure $3 a$ and $b$. The projections of the hydrogels with MA modification show that the hydrogels are less transparent suggesting that MA changes their optical properties and in consequence its texture. In addition, photoGG-MA and iGG-MA projection images look similar to the naked eye. However, photoGG-MA hydrogel is cross-linked by covalent bonding and later stabilized by immersion in PBS; thus, these hydrogels are also physically cross-linked by ionic interaction. Thus, it is expected a stronger cross-linking for photoGG-MA, due to the combination of the cross-linking methods, compared to the iGG-MA, which is ionically cross-linked. In addition, the MA-modification in iGGMA affect the amount of available carboxyl groups and thus has less available cross-linking sites when compared to $\mathrm{iGG}$, which 


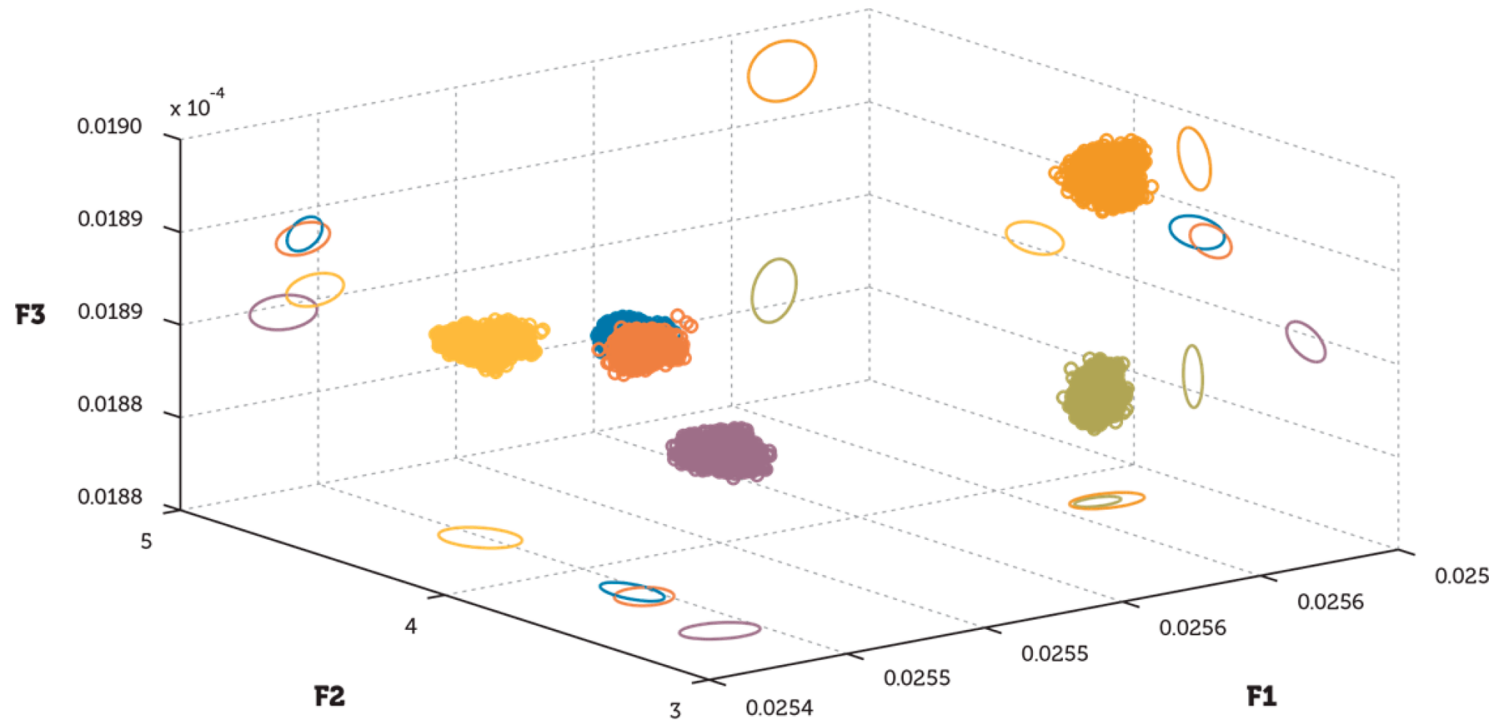

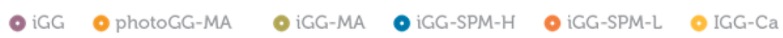

(a)

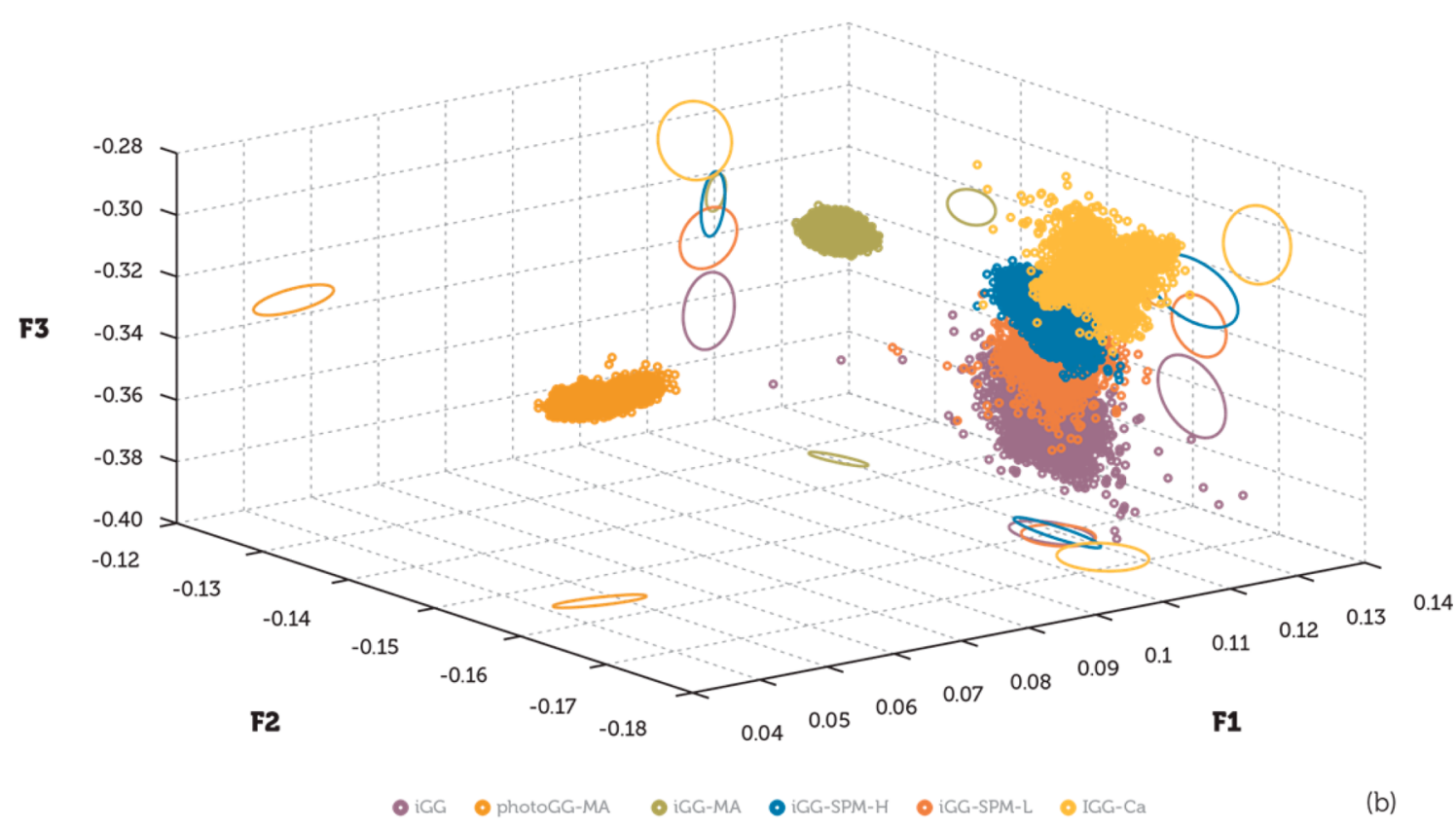

Figure 4. Three-dimensional distributions of the six types of hydrogels projected onto 2D subspaces: (a) projections and (b) reconstructions. iGG, ionic cross-linked gellan gum; iGG-MA, ionic cross-linked methacrylated gellan gum; photoGG-MA, photo-cross-linked methacrylated gellan gum; iGG-SPM-L, bioamine low cross-linked gellan gum; iGG-SPM-H, bioamine high cross-linked gellan gum; and iGG-Ca, calcium ions cross-linked gellan gum.

corresponds to physically cross-linked non modified gellan gum. Therefore, this could cause differences in the cross-linking density and distribution. These differences promote a change in the microstructure of the hydrogel and thus in the image texture of these hydrogels, which are possible to evaluate through the use of OPT and MDA. In addition, in previous studies Silva-Correia et al. ${ }^{35}$ have reported that phGG-MA has a storage modulus higher than iGG hydrogels and iGG-MA shows an intermediate behavior; therefore this method can be used to predict some properties of different formulations of hydrogels.

Moreover, image textural analysis can find structural differences between these types of hydrogels. This can be seen from the results obtained from the reconstructed slices. The reconstruction gives information about the internal structure of the hydrogel; thus, the use of the reconstructed slices enables the possibility to analyze a different dimension than the provided by the projections. MDA results show a clear separation between these groups of hydrogels in the projection images and reconstructed slices, suggesting that OPT can detect cross-linking density, distribution, and method and discriminate between modified and nonmodified GG.

Second group includes physical cross-linking GG hydrogels. In this group, two of the hydrogels are cross-linked by the bioamine spermine (iGG-SPM-H and iGG-SPM-L) and the third is cross-linked by $\mathrm{Ca}^{2+}$ cation (iGG-Ca). Polymer 

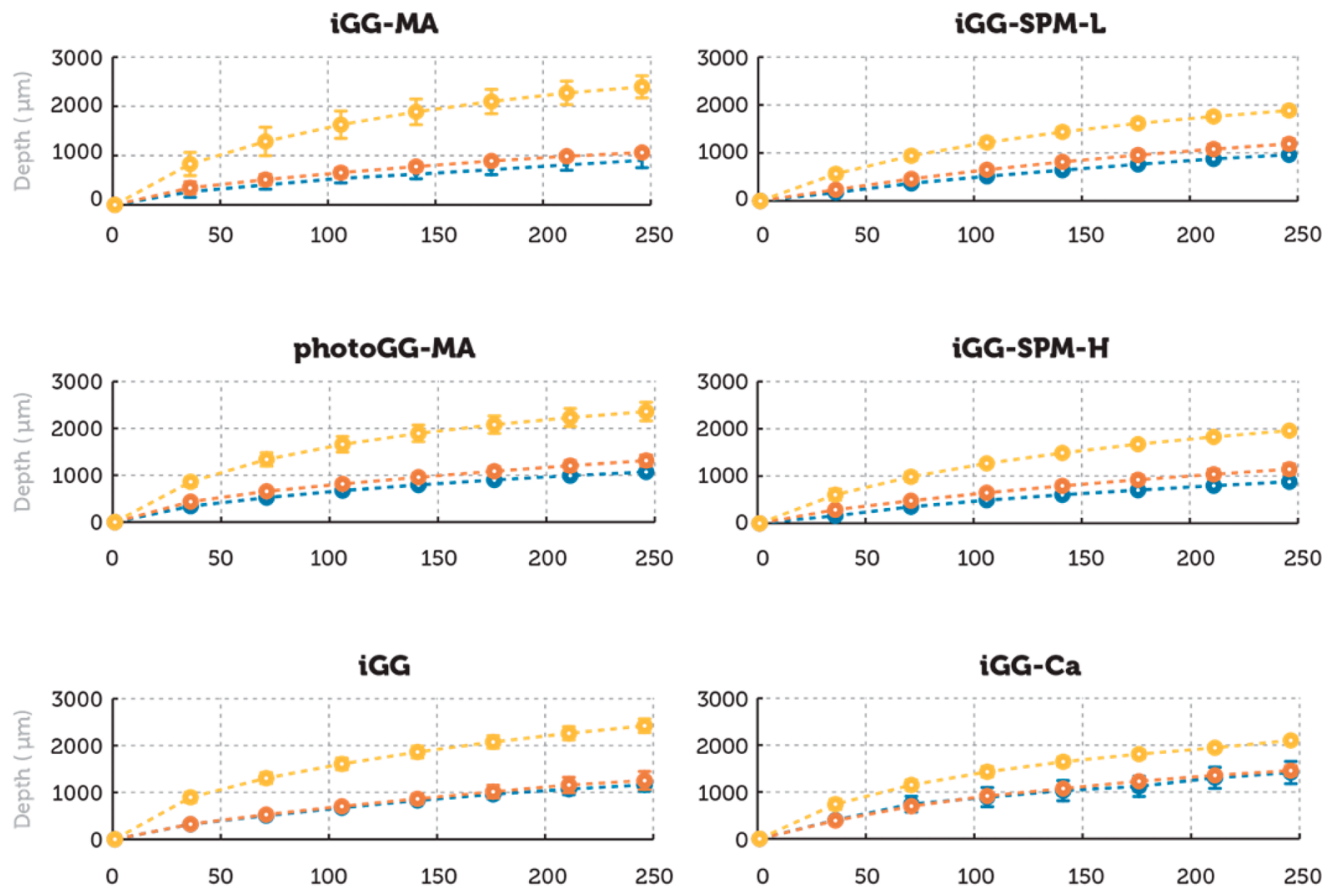

Time (min)

$\longmapsto-12000 \mathrm{kDa} \longmapsto-150 \mathrm{kDa} \longmapsto-120 \mathrm{kDa}$ Time (min)

Figure 5. Progression of the frontwave of the binary images. The curves represent the median of the five samples and the vertical bars represent the standard deviation. iGG, ionic cross-linked gellan gum; iGG-MA, ionic cross-linked methacrylated gellan gum; photoGG-MA, photo-cross-linked methacrylated gellan gum; iGG-SPM-L, bioamine low cross-linked gellan gum; iGG-SPM-H, bioamine high cross-linked gellan gum; and iGG-Ca, calcium ions cross-linked gellan gum.

concentration and cross-linking method (physical cross-linking) is the same in this group of hydrogels, however the concentration of cross-linking agent varies.

From MDA results, it can be seen that iGG-SPM-H and iGG-SPM-L clusters are closer in the MDA of the reconstructed slices and in the projection images. Despite the fact that these hydrogels are similar, since the only difference is the cross-linker concentration, it is possible to appreciate that the features clusters are closer but still separated, which indicates small differences between them. From previous studies, Koivisto et al. ${ }^{29}$ has found out that iGG-SPM-L has lower young modulus than iGG-SPM-H which is expected from the different cross-linking concentration. On the other hand, there is a clear separation between the hydrogels cross-linked with $\mathrm{Ca}^{2+}$ and SPM. The bioamine SPM is a tetravalent salt and $\mathrm{CaCl}_{2}$ is divalent. Therefore, the hydrogels produced by $\mathrm{Ca}^{2+}$ cross-linking are expected to be structurally slightly different from the hydrogels produced with SPM. This result indicates that OPT can also detect structural differences caused by crosslinking concentration, cross-linking density and cross-linking agent, which ultimately affects the hydrogel properties such as mechanical characteristics,

Figure 4 shows the results of the MDA of the two groups of hydrogels together. In this case, we chose to reduce to three dimensions with three discriminant functions (F1, F2, and F3). The different hydrogel classes were projected onto twodimensional subspaces for better visualization. The functions for the projections and reconstructed slices are built again from similar combination of texture features as described before.

From the MDA of the two groups together (Figure 4) it is possible to observe that the separation between modified gellan gum (GG-MA) and nonmodified GG is evident. The nonmodified gellan gum hydrogels classes are closer together in both projections and reconstructed slices suggesting that with transmission OPT is possible to detect differences between the types of polymers. Furthermore, the most similar hydrogels (i.e., iGG-SPM-H and iGG-SPM-L) maintain their closeness and separation with the other groups, iGG and iGG$\mathrm{Ca}$ in both the projections and reconstructed slices. The separation between iGG and iGG-Ca groups could be due to the difference in polymer concentration or cross-linking density; however, it is possible to notice that there is an evident difference between these two groups. Finally, iGG-MA and photoGG-MA are much more separated from the nonmodified hydrogel groups, and there is still a separation between these modified gellan gum groups. The results suggest that the cross-linking method has an effect in the final structure of these hydrogels.

The results demonstrate that imaging with transmission OPT together with texture analysis and statistical methods, form a tool that is able to characterize tissue engineering hydrogels during the design and fabrication phases. Moreover, this can be done in a hydrated condition which is closer to the in vivo environment. Transmission OPT allows us to inspect the hydrogel structure not only in one dimension (i.e., the projections images), but also in the third dimension with reconstructed slices, and asses the internal structural changes in the hydrogel due to different compositions. Therefore, we consider that the degree and efficiency of hydrogel cross-linking can be assessed with transmission mode OPT, and this is an important factor when designing hydrogels, since it affects their physical properties and performance. ${ }^{36}$ This technique can be used to reduce time and the amount of samples prepared 


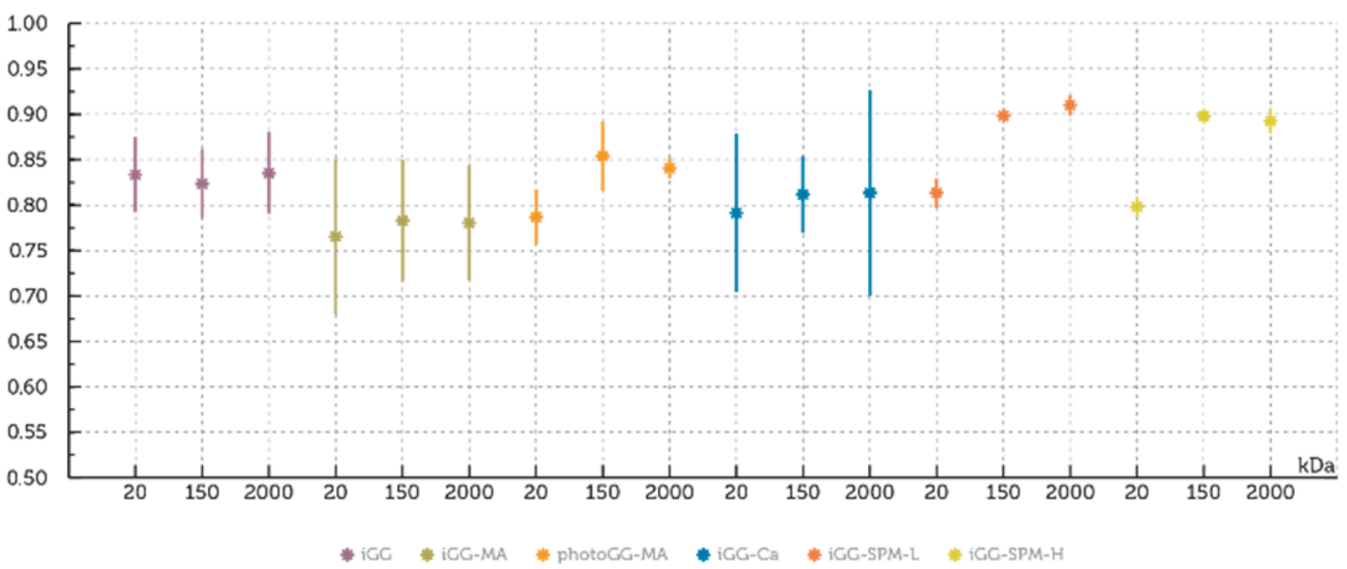

Figure 6. Index of homogeneity. iGG, ionic cross-linked gellan gum; iGG-MA, ionic cross-linked methacrylated gellan gum; photoGG-MA, photocross-linked methacrylated gellan gum; iGG-SPM-L, bioamine low cross-linked gellan gum; iGG-SPM-H, bioamine high cross-linked gellan gum; and iGG-Ca, calcium ions cross-linked gellan gum.

during the design of hydrogels and to predict hydrogel suitability for a specific application.

3.2. Mass Transport Studies. Generally, in tissue engineered hydrogel scaffolds, molecules travel though the water filled hydrogel mesh and they rely in passive diffusion since no vasculature exists. Thus, the cross-linking density and inhomogeneity of the cross-linking distribution, size of the porous and porous interconnectivity are some of the factors affecting the transportation of molecules in hydrogels. ${ }^{37,38}$ The mobility of differently sized molecules depends on the crosslink density and hydrogel mesh, allowing or restricting their transport. ${ }^{38}$ Thus, in this work, mass transport of molecules was studied with emission OPT to examine their mobility through the three-dimensional structure of hydrogels. For this, FITCdextrans were used with different molecular weights (i.e., 20, 150 , and $2000 \mathrm{kDa}$ ) as model molecules, comparable to nutrients (glucose, $0.18 \mathrm{kDa}$ ), proteins (fibronectin, $550 \mathrm{kDa}$; TGF- $\beta, 25 \mathrm{kDa}$; proteoglycans, $2500 \mathrm{kDa}$ ), waste, and other metabolites. $^{39}$

The median value of the progression of the wavefront in the binary images corresponding to the FITC-dextran traveling downward through the hydrogel is plotted in Figure 5. The results showed that the $20 \mathrm{kDa}$ FITC-dextran travels faster and deeper through the hydrogel. On the other hand, 150 and 2000 $\mathrm{kDa}$ have a similar velocity and do not travel as deep into the gel during the time of the measurement. It was also found that the velocities in all the measurements are not constant. At the beginning the velocities are faster and get slower when going deeper into the hydrogel (see Figure S1 in the Supporting Information). This might be due to the reduction of the dextran concentration on the top of the hydrogel over time as dextran diffuses inside.

Mann-Whitney $\mathrm{U}$ test revealed statistically significant ( $p<$ 0.05 ) differences for most of the comparisons between different FITC-dextran molecular weights traveling in the same type of hydrogel, except when comparing $2000 \mathrm{kDa}$ with $150 \mathrm{kDa}$ in iGG-Ca, photoGG-MA, and iGG. From Figure 5, it can be seen that the progression curves are similar for these two molecular weights, and this can be due to $150 \mathrm{kDa}$ has a threshold size which Stokes' radii is between 69 and $95 \AA \AA^{40}$ above which the hydrogel mesh affects the transport, Stokes' radii of $2000 \mathrm{kDa}$ is $270 \AA \AA^{40}$ Furthermore, when comparing the curves for different types of hydrogels with the same FITC-dextran molecular weights, no statistical difference was found for iGG-SPM-L,
iGG-SPM-H, and photoGG-MA. This may be due to the fact that these hydrogels present similar pore size. Large standard deviation is produced by inhomogeneity of the samples and affects the statistical differences. These results are consistent with previous findings. Silva-Correia et al. ${ }^{35}$ reported that iGGMA and photoGG-MA prevented the infiltration of endothelial cells and blood vessels due to their compact structure, which makes these hydrogels good candidates for nucleus pulposus applications. Koivisto et al. $^{29}$ studied transportation of molecules on iGG-SPM-L and iGG-SPM-H with Fluorescence recovery after photobleaching (FRAP) technique finding no differences in transportation between these hydrogels.

Figure 6 shows the results of the index of homogeneity with different FITC-dextran molecular weights, for all the hydrogels (the wavefront traveling downward in the binary images is shown in Supporting Information Videos 2 and 3). From the results, it was observed that iGG-SPM-L and iGG-SPM-H are the most homogeneous hydrogels for all the molecules and with smaller standard deviation. The statistical difference of the computed indexes of homogeneity between different FITCdextrans molecular weights, traveling in the same type of hydrogel and between different types of hydrogels with the same FITC-dextrans molecular weights, was also tested. Statistical differences were found for iGG-SPM-L with different FITC-dextrans molecular weights. There was no statistical differences in the indexes of homogeneity for the progression of $20 \mathrm{kDa}$ molecule in the different types of hydrogels, because the hydrogel mesh does not hinder their mobility considering that their Stokes' radii is between 24 and $45 \AA^{40}{ }^{40}$ For the other two FITC-dextrans molecular weights, statistical differences were found between iGG-SPM-L, iGG-Ca, photoGG-MA, and iGG, and between iGG-SPM-H, photoGG-MA, and iGG.

The higher standard deviation and smaller values of homogeneity in iGG-MA hydrogels when compared to iGG can be attributed to the differences in sample preparation. iGG hydrogels are prepared by mixing the solution with PBS, while iGG-MA hydrogels are not mechanically mixed with PBS, but the PBS is slowly poured on top of the GG-MA gel solution for overnight stabilization. This might cause less homogeneous distribution of the ions in the solution, producing inhomogeneous cross-linking distribution. Thus, the method for preparation and time of stabilization can have an impact in the homogeneity of the hydrogel. On the other hand, due to the methacrylation of GG backbone, the amount of available 
carboxyl groups for ionic interaction in iGG-MA hydrogels is lower than that in native GG. So the chemical modification of GG can influence the cross-linking process in both iGG-MA and photoGG-MA hydrogels.

Furthermore, the higher standard deviation in iGG-Ca hydrogels, Figure 6, can be attributed to more variability between samples due to the method of preparation and their fast gelation time, i.e., approximately in seconds. iGG-SPM-L and $\mathrm{iGG}-\mathrm{SPM}-\mathrm{H}$ have a longer gelation time which makes the mixing between polymer solution and cross-linker solution more efficient. As a result, the produced hydrogels with this concentration of bioamine are more consistent, which is apparent from the smaller standard deviation presented in Figure 6. Thus, with this method it is also possible to assess the repeatability of the method of preparation.

The results show that the index of homogeneity is useful in studying how freely the molecules can travel through the hydrogels, as well as the cross-linking distribution, which contribute to the homogeneity of the hydrogel structure. Thus, this index can be used as one of the parameters to be correlated to cell response. Even though the mass transport method presented here does not give a precise number for pore size, this technique is able to determine the molecular mobility within the hydrogel, which can be even more relevant for designing tissue engineered hydrogels than just pore size. ${ }^{21}$ However, the transport behavior might change when cells produce ECM while the tissue is developing and/or hydrogel is degrading. ${ }^{37,38}$ As our method is nondestructive, it presents the possibility to track those changes in transparent hydrogels in the presence of cells and understand the changes during time in mass transport.

\section{CONCLUSION}

In this work, the capabilities of using optical methods based in OPT technique to analyze material characteristics of hydrated hydrogels were demonstrated. Through visual inspection of hydrogels with OPT it is possible to monitor changes in image texture according to the modifications during the formulation of hydrogels, such as cross-linking concentration or polymer modification.

The usefulness of OPT to characterize mass transport in macroscopic hydrogel scaffold was validated using a model molecule in order to mimic the transport of nutrients and other biomolecules relevant for tissue engineering. Also, an index of homogeneity was defined to evaluate the cross-linking density and distribution. This can be further used to control the efficiency and repeatability of the hydrogel production.

The methods presented here allow the possibility of screening hydrogel compositions. Different groups of hydrogels can be analyzed and compared against a group of hydrogels with known and desired properties. It is expected that with a known cell response to certain hydrogel composition/structure, one can correlate it to the desired features of the newly designed hydrogels. Therefore, with this kind of screening and correlation the amount of cell testing needed can be reduced in the future.

Due to their low cost of components, low time consumption for imaging, and easiness of the method, we believe that OPT system offers good capabilities to develop methods for characterization of hydrogels. The main advantage of OPT is the possibility to characterize hydrogels in the exact same state they will be in the final application, i.e., in the wet form. We showed that the set of OPT imaging based material characterization methods we developed here are useful for screening many characteristics of hydrogel compositions in relatively short time in an inexpensive manner. The set of methods developed in this work provide new tools for hydrogel development and has potential to impact the design, development, and enhancement of hydrogels for tissue engineering and drug/cell delivery applications.

\section{ASSOCIATED CONTENT}

\section{S Supporting Information}

The Supporting Information is available free of charge on the ACS Publications website at DOI: 10.1021/acs.langmuir.6b00554.

Video 1 shows examples of projections and reconstructions of two different types of hydrogels: (a) shows iGGSPM-H projections, (b) the reconstruction of (a), (c) photoGG-MA projections, and (d) reconstruction of (c). Video 2 shows the wavefront traveling downward in the binarized images. Video 3 shows an inhomogeneous wavefront in the binarized images. Figure S1 shows FITC-dextran velocities in hydrogels at different time intervals. (ZIP)

\section{AUTHOR INFORMATION}

\section{Corresponding Authors}

*E-mail: ana.sotodelacruz@gmail.com.

*E-mail: jari.hyttinen@tut.fi.

\section{Notes}

The authors declare no competing financial interest.

\section{ACKNOWLEDGMENTS}

The authors thank Tekes, Jane and Aatos Erkko Foundation, and EXTREMA COST Action MP1207 for supporting this work. J.S.-C. would like to acknowledge the Portuguese Foundation for Science and Technology (FCT; Grant No. SFRH/BPD/100590/2014). The authors would also like to thank Ibrahim Fatih Cengiz for his assistance in the production of methacrylated gellan gum.

\section{REFERENCES}

(1) Langer, R.; Vacanti, J. P. Tissue Engineering. Science 1993, 260, 920-926.

(2) Griffith, L. G.; Naughton, G. Tissue Engineering-Current Challenges and Expanding Opportunities. Science 2002, 295, 10091014.

(3) Slaughter, B. V.; Khurshid, S. S.; Fisher, O. Z.; Khademhosseini, A.; Peppas, N. A. Hydrogels in Regenerative Medicine. Adv. Mater. 2009, 21, 3307-3329.

(4) Geckil, H.; Xu, F.; Zhang, X.; Moon, S.; Demirci, U. Engineering Hydrogels as Extracellular Matrix Mimics. Nanomedicine (London, U. K.) 2010, 5, 469-484.

(5) Jin, R.; Dijkstra, P. J. Biomedical Applications of Hydrogels Handbook. In Biomedical Applications of Hydrogels Handbook; Ottenbrite, R. M., Park, K., Okano, T., Eds.; Springer: New York, 2010; pp 203-225.

(6) Teßmar, J.; Brandl, F.; Göpferich, A. Hydrogels for Tissue Engineering. In Fundamentals of Tissue Engineering and Regenerative Medicine; Meyer, U., Handschel, J., Wiesmann, H. P., Meyer, T., Eds.; Springer: Berlin, Heidelberg, 2009.49510.1007/978-3-540-77755737

(7) Van Vlierberghe, S.; Dubruel, P.; Schacht, E. Biopolymer-Based Hydrogels as Scaffolds for Tissue Engineering Applications: A Review. Biomacromolecules 2011, 12, 1387-1408. 
(8) Drury, J. L.; Mooney, D. J. Hydrogels for Tissue Engineering: Scaffold Design Variables and Applications. Biomaterials 2003, 24, 4337-4351.

(9) Silva-Correia, J.; Oliveira, J. M.; Caridade, S. G.; Oliveira, J. T.; Sousa, R. A.; Mano, J. F.; Reis, R. L. Gellan Gum-Based Hydrogels for Intervertebral Disc Tissue-Engineering Applications. J. Tissue Eng. Regener. Med. 2011, 5, e97-e107.

(10) Pereira, D. R.; Silva-Correia, J.; Oliveira, J. M.; Reis, R. L. Hydrogels in Acellular and Cellular Strategies for Intervertebral Disc Regeneration. J. Tissue Eng. Regener. Med. 2013, 7, 85-98.

(11) Silva-Correia, J.; Pereira, H.; Yan, L. P.; Miranda-Gonçalves, V.; Oliveira, A. L.; Oliveira, J. M.; Reis, R. M.; Espregueira-Mendes, J. D.; Reis, R. L. Advanced Mimetic Materials for Meniscus Tissue Engineering: Targeting Segmental Vascularization. J. Tissue Eng. Regen. Med. 2012, 6, 8-39.

(12) Pereira, H.; Silva-Correia, J.; Yan, L. P.; Caridade, S. G.; Frias, A. M.; Oliveira, A. L.; Mano, J. F.; Oliveira, J. M.; Espregueira-Mendes, J.; Reis, R. L. Silk-Fibroin/Methacrylated Gellan Gum Hydrogel as an Novel Scaffold for Application in Meniscus Cell-Based Tissue Engineering. Arthrosc. J. Arthrosc. Relat. Surg. 2013, 29, e53-e55.

(13) Haastert-Talini, K.; Geuna, S.; Dahlin, L. B.; Meyer, C.; Stenberg, L.; Freier, T.; Heimann, C.; Barwig, C.; Pinto, L. F. V; Raimondo, S.; et al. Chitosan Tubes of Varying Degrees of Acetylation for Bridging Peripheral Nerve Defects. Biomaterials 2013, 34, 98869904.

(14) Hunt, J.; Chen, R.; Van Veen, T.; Bryan, N. Hydrogels for Tissue Engineering and Regenerative Medicine. J. Mater. Chem. B 2014, 2, 5319-5338.

(15) Liu, J.; Hilderink, J.; Groothuis, T. A. M.; Otto, C.; van Blitterswijk, C. A.; de Boer, J. J. Tissue Eng. Regener. Med. 2015, 9, 952-960.

(16) Stevens, M. M.; George, J. H. Exploring and Engineering the Cell Surface Interface. Science 2005, 310, 1135-1138.

(17) Amsden, B. Solute Diffusion within Hydrogels. Mechanisms and Models. Macromolecules 1998, 31, 8382-8395.

(18) Amsden, B. Solute Diffusion in Hydrogels. An Examination of the Retardation Effect. Polym. Gels Networks 1998, 6, 13-43.

(19) Okay, O. General Properties of Hydrogels. In Hydrogel Sensors and Actuators; Gerlach, G., Arndt, K.-F., Eds.; Springer Series on Chemical Sensors and Biosensors; Springer: Berlin, Heidelberg, 2010; Vol. 6, pp 1-14.

(20) Vielreicher, M.; Schürmann, S.; Detsch, R.; Schmidt, M. A.; Buttgereit, A.; Boccaccini, A.; Friedrich, O. Taking a Deep Look: Modern Microscopy Technologies to Optimize the Design and Functionality of Biocompatible Scaffolds for Tissue Engineering in Regenerative Medicine. J. R. Soc., Interface 2013, 10, 20130263.

(21) Mather, M. L.; Morgan, S. P.; Crowe, J. A. Meeting the Needs of Monitoring in Tissue Engineering. Regener. Med. 2007, 2, 145-160.

(22) Chalal, M.; Morfin, I.; Vial, J.; Aguilar de Armas, M. A.; San Roman, J. S.; Bolgen, N.; Piskin, E.; Ziane, O.; Casalegno, R. Macromolecules 2009, 42, 2749-2755.

(23) Sharpe, J.; Ahlgren, U.; Perry, P.; Hill, B.; Ross, A.; HecksherSørensen, J.; Baldock, R.; Davidson, D. Optical Projection Tomography as a Tool for 3D Microscopy and Gene Expression Studies. Science 2002, 296, 541-545.

(24) Sharpe, J. Optical Projection Tomography as a New Tool for Studying Embryo Anatomy. J. Anat. 2003, 202, 175-181.

(25) Rieckher, M.; Birk, U. J.; Meyer, H.; Ripoll, J.; Tavernarakis, N. Microscopic Optical Projection Tomography In Vivo. PLoS One 2011, 6, e18963.

(26) Soto, A. M.; Koivisto, J.; Parraga, J. E.; Silva-Correia, J.; Oliveira, J. M.; Reis, R. L.; Kellomäki, M.; Hyttinen, J.; Figueiras, E. Optical Projection Tomography as a Tool for Visualizing Hydrogels Microstructures. In 26th Annual Conference of the European Society for Biomaterials (ESB); 2014.

(27) Figueiras, E.; Soto, A. M.; Jesus, D.; Lehti, M.; Koivisto, J.; Parraga, J. E.; Oliveira, J. M.; Reis, R. L.; Kellomäki, M.; Hyttinen, J. Optical Projection Tomography as a Tool for 3D Imaging of Hydrogels. Biomed. Opt. Express 2014, 5, 3443-3449.
(28) Soto, A. M.; Koivisto, J.; Parraga, J. E.; Hyttinen, J.; Kellomäki, M.; Figueiras, E. Mass Transport Study with Fluorescent Dextran Molecules in Gellan Gum Hydrogel. In 27th Annual Conference of the European Society for Biomaterials (ESB); 2015.

(29) Koivisto, J.; Teymouri, S.; Parraga, J. E.; Ihalainen, T. O.; AaltoSetälä, K.; Kellomäki, M. Development of Bioamine Cross-Linked Gellan Gum Hydrogels as Soft Scaffolds for Neural Tissue Engineering. In 26th Annual Conference of the European Society for Biomaterials (ESB); Liverpool, 2014; pp 39-5.

(30) Silva-Correia, J.; Gloria, A.; Oliveira, M. B.; Mano, J. F.; Oliveira, J. M.; Ambrosio, L.; Reis, R. L. Rheological and Mechanical Properties of Acellular and Cell-Laden Methacrylated Gellan Gum Hydrogels. J. Biomed. Mater. Res., Part A 2013, 101, 3438-3446.

(31) Haralick, R. M.; Shanmugam, K.; Dinstein, I. Textural Features for Image Classification. IEEE Trans. Syst. Man. Cybern. 1973, 3, 610.

(32) Duda, R. O.; Hart, P. E.; Stork, D. G. Pattern Classification; John Wiley \& Sons: New York, 2001.

(33) Gonzalez, R. C.; Woods, R. E. Digital Image Processing, 3rd ed.; Prentice Hall: Upper Saddle River, NJ, 2008.

(34) Jain, R; Kasturi, R; Schunck, B. G. Machine Vision; Munson, E. M., Ed.; McGraw-Hill: New York, 1995.

(35) Silva-Correia, J.; Zavan, B.; Vindigni, V.; Silva, T. H.; Oliveira, J. M.; Abatangelo, G.; Reis, R. L. Biocompatibility Evaluation of Ionicand Photo-Crosslinked Methacrylated Gellan Gum Hydrogels: In Vitro and In Vivo Study. Adv. Healthcare Mater. 2013, 2, 568-575.

(36) ASTM F2900-11, Standard Guide for Characterization of Hydrogels Used in Regenerative Medicine; ASTM International: West Conshohocken, PA, 2011.

(37) Watkins, A. W.; Anseth, K. S. Investigation of Molecular Transport and Distributions in Poly(ethylene Glycol) Hydrogels with Confocal Laser Scanning Microscopy. Macromolecules 2005, 38, 13261334.

(38) Dhote, V.; Skaalure, S.; Akalp, U.; Roberts, J.; Bryant, S. J.; Vernerey, F. J. On the Role of Hydrogel Structure and Degradation in Controlling the Transport of Cell-Secreted Matrix Molecules for Engineered Cartilage. J. Mech. Behav. Biomed. Mater. 2013, 19, 61-74.

(39) Leddy, H. A.; Guilak, F. Site-Specific Molecular Diffusion in Articular Cartilage Measured Using Fluorescence Recovery after Photobleaching. Ann. Biomed. Eng. 2003, 31, 753-760.

(40) FITC-Dextran Fluorescein Isothiocyanate Dextran; http:// tdbcons.com/images/pdf/fitcdextran2.pdf.

(41) Szczesniak, A. S. Texture Is a Sensory Property. Food Qual. Prefer. 2002, 13, 215-225. 